\title{
Benchmarking e a indústria do conhecimento: um estudo de caso
}

Adalberto Rego Maciel Filho

Professor Titular da Faculdade de Ciências da Administração de Pernambuco - Universidade de Pernambuco.Mestre em Ciência Regional University of Pennsylvania.Doutor em Economia - New School for Social Research New York

Miriam Cunha de Aquino

\begin{abstract}
Especialista em Documentação Científica Consultora/Pesquisadora - Departamento de Ciência da Informação da Universidade Federal de Pernambuco/UFPE

(aposentada).
\end{abstract}

Nayade Kennedy Sales

http://dx.doi.org/10.1590/1981-5344/2730

Estudo de caso das Bibliotecas Indústria do Conhecimento (IDCs) do sistema do Serviço Social da Indústria-Sesi localizadas em Alagoas (25), Paraíba (10), Pernambuco (18), Rio Grande do Norte (10), especificamente as IDCs Públicas, objetivando analisar o seu desempenho e apresentar oportunidades para incremento de frequência utilizando benchmarking. Inclui revisão de literatura de projetos nacionais de uso de benchmarking, bem como estudos e considerações sobre esse processo. Para análise dos dados, criaram-se dois índices: o de alcance municipal e o de eficiência, que relacionam as frequências às IDCs à população em idade escolar (PIE) de cada município e com horas de funcionamento mensais respectivamente. Sugerem-se oito etapas para execução de uma política de benchmarking para as IDCs fundamentada nas precondições existentes no sistema composto de 326 unidades onde, aproximadamente, 275 são públicas. Política que poderia ser adotada para as bibliotecas públicas municipais. 
Palavra chave: Biblioteca Indústria do Conhecimento; Benchamarking; Políticas públicas.

\title{
Benchmarking and the knowledge industry: a case study
}

\begin{abstract}
Case study of the knowledge Industry Libraries (IDCs) owned by the Industrial Social Service System (Sesi) located in Alagoas (25), Paraíba (10), Pernambuco (18), Rio Grande do Norte (10), specifically the ones located in public areas, aiming to investigate their performance and present opportunities to increase attendance by using benchmarking. It includes a literature review of national projects that use benchmarking, as well as, other studies that discuss and examine this procedure. In order to analyze the data, two indices were created: the municipal scope and efficiency, relating the attendance to IDCs to the population at school age (PIE) of each municipality and to monthly working hours respectively. Eight stages are suggested to perform a benchmarking policy for IDCs based on existing preconditions in the system composed by approximately 275 units. This policy could be also applied to the municipal public library system.
\end{abstract}

Keywords: Knowledge Industry Libraries; Benchamarking; Public policy.

Recebido em 03.02.2016 Aceito em 17.05.2016

\section{Introdução}

O objetivo deste trabalho ${ }^{1}$ é analisar o desempenho comparativo das Bibliotecas Indústria do Conhecimento (IDCs) localizadas nos estados das Alagoas (AL), da Paraíba (PB), de Pernambuco (PE) e do Rio Grande do Norte (RN), particularmente as IDCs Públicas, pertencentes ao sistema Serviço Social da Indústria (Sesi), e as oportunidades de crescimento decorrentes da utilização do benchmarking.

A técnica de benchmarking foi, originalmente, desenvolvida no setor privado, pela Xerox nos anos 70 do século passado e, posteriormente, a partir da primeira metade da década de 90 , com a grande divulgação dos programas de qualidade, passou a ser, também, utilizada pelo setor público em vários países. (FAVRET, 2000).

\footnotetext{
1 Nesse projeto trabalharam as seguintes bolsistas financiadas pelo fundo de pesquisa FCAP-UPE: Mariane Araújo, Nayade Sales, Beatriz Cavalcanti, Gabriela Silveira e Letícia Silveira.
} 
Fenna e Knüpling (2012) ilustram essa técnica em estudo composto de artigos relativos às experiências com benchmarking no setor público de diversos países investigados. Por exemplo, analisa no programa americano No Children Left Behind, no sistema de saúde do Canadá, no desenvolvimento sustentável na Suíça e em diversos setores públicos na Austrália. Knüpling (2012, p. 4) destaca: "Alemanha, [...] é única no sentido que fez uma provisão constitucional para o benchmarking, na oferta dos serviços públicos para o país, em recente emenda constitucional, no contexto da maior reorganização do federalismo alemão." O autor ressalta, no entanto, que a aplicação do benchmarking na Alemanha ainda se encontra em fase de implantação, ou seja, na infância. Para maiores detalhes vide Konzendorf e Hathawa (2012).

A segunda parte deste trabalho é uma revisão da literatura dos projetos e práticas de benchmarking de diversos países, incluindo estudos realizados com amostras menores e projetos especiais. O estudo de caso relativo às Bibliotecas Indústria do Conhecimento compõe a terceira. Na última parte, estão às considerações finais.

\section{Revisão de experiências nacionais na utilização do benchmarking}

Observou-se, nas análises das experiências de alguns países, que o processo de aplicação do benchmarking nos sistemas de bibliotecas públicas desenvolveu-se em aproximadamente quatro etapas: elaboração de indicadores de desempenho; análise das informações coletadas; elaboração da estratificação/ ranqueamento; e transferências de experiências/informações. Constatou-se, também, a importância das precondições para o processo de benchmarking ser utilizado: um sistema de coleta de dados, análise e divulgação das estatísticas com regularidade.

$\mathrm{Na}$ discussão dos estudos de casos, tentou-se seguir as quatro etapas mencionadas, sempre que possível.

\subsection{Revisão de literatura de projetos nacionais}

Apresentam-se a seguir informações sobre os projetos nacionais alemão, norte-americano, norueguês, sueco, dinamarquês e de outros países.

\subsubsection{Alemanha}

Mundt (2009, p. 188) afirma que um dos primeiros resultados do processo sistemático de benchmarking na Alemanha apareceu em 1999, e compreendeu 85 bibliotecas acadêmicas e 170 públicas. Por sua vez, foi fundamentado em estudo inicial realizado pela Bertelsmann Foundation 
com 18 bibliotecas públicas. ${ }^{2}$ (PRÖHL; WINDAU, 1997a; 1997b apud MUNDT, 2009, p. 188). "O Projeto foi baseado na percepção de que as comparações interinstitucionais poderiam substituir a falta de competição do setor público e ser catalisador apropriado para o aperfeiçoamento contínuo."

O sistema denominado Bix classifica as bibliotecas utilizando o critério de uma a quatro estrelas (quatro estrelas significam o melhor desempenho) em quatro grupos de indicadores: serviços, uso, eficiência e desenvolvimento, compostos por seis, cinco, quatro e três, respectivamente, disponíveis no site do Bix.

Os indicadores para serviços são: unidades de coleção per capita ; espaço do usuário no piso em $\mathrm{m}^{2}$ per 1.000 capita; empregados per 1.000 capita; estação de computadores per 1.000 capita; serviços de internet; e eventos per 1.000 capita.

Uso: visitas per capita; visitas virtuais per capita; empréstimos per capita; taxa de turnover dos estoques; total de horas abertas ao público anual per 1.000 capita; e número de pontos de serviços.

Eficiência: orçamento para aquisição por empréstimo; empregados por horas abertas ao público; visitas por horas abertas; e despesas correntes por visitas (os indicadores são ranqueados em ordem inversa: menor valor significa melhor valor).

Desenvolvimento: taxa de renovação; treinamento avançados por empregado; e investimento em capital por empregado.

Depois de nove anos de utilização do Bix, tornou-se um sucesso colocar as bibliotecas na agenda/evidência e encorajar inciativas de qualidade e benchmarking entre as bibliotecas públicas e acadêmicas. 0 Bix tornou-se, crescentemente, autodinâmico nas bibliotecas, no sentido de os tomadores de decisão e fundações financiadoras imporem uma forte demanda nas bibliotecas para 0 desenvolvimento de um instrumento mais aperfeiçoado e a introdução de medidas de processos de qualidade, valor, impacto e resultados." (MUNDT, 2009, p. 194).

Hoje o Bix analisa dados de 2.000 bibliotecas públicas e 240 acadêmicas. "Tem como princípio básico que as bibliotecas participem voluntariamente. Cada biblioteca decide se quer que seus dados sejam publicados ou não". Desde 2005, a Associação Alemã de Bibliotecas e o North Rhine-Westphalian Library Service Center o divulgam (ASSOCIAÇÃO ALEMÃ DE BIBLIOTECAS; NORTH RHINE-WESTPHALIAN LIBRARY SERVICE CENTER ).

Analisando o crescimento das bibliotecas públicas participantes, de 170 unidades em 1999 para 2.000, no ano de 2015, e levando-se em consideração a participação voluntária no processo, é razoável concluir que os resultados publicados devem ter sido bastante utilizados/úteis na gestão das respectivas bibliotecas públicas.

\footnotetext{
${ }^{2}$ PRÖHL, M.; WINDAU, B. (Eds.). Betriebsvergleich an Öffentlichen Bibliotheken. Teil 1: Empfehlungen und Arbeitsmaterialien für ein output-orientiertes Berichtswesen. Gütersloh, 1997a. v.1.

PRÖHL, M.; WINDAU, B. (Eds.). Betriebsvergleich an Öffentlichen Bibliotheken. Teil 2: Meßergebnisse Richtwerte - Handlungsempfehlungen. Gütersloh, 1997b. v.2.
} 


\subsubsection{Estados Unidos}

O índice do Library Journal (LJ) e o sistema de classificação começaram a ser publicados em 2008 nos Estados Unidos. Lyons e Lance (2014, p. 22) afirmam:

a esperança era de que se as bibliotecas fossem premiadas ou não, elas examinassem as estatísticas de perto - para sua própria biblioteca e dos seus pares - e fizessem uso dessas estatísticas e de outros tipos de dados com o propósito de planejamento local e avaliação.

O LJ baseia-se em quatro tipos de indicadores per capita calculados pela própria equipe: visitas, circulação, acesso/uso do público ao computador e atendimento a programas/eventos; são separados em nove faixas de acordo com a capacidade de gastos/orçamento. Para facilitar a comparação criou-se um sistema de estrelas que identificam as melhores bibliotecas (cinco a uma estrela) seguindo a lógica do modelo alemão. O número de bibliotecas analisadas nos Estados Unidos está aumentando. Em 2009, eram 7.115 unidades e, em 2014, 7.586. (LYONS; LANCE, 2014, p. 28). Houve um incremento de 6\%.

$\mathrm{O}$ LJ Index fundamenta-se nos dados compilados anualmente, desde 1988, pelo Institute of Museum and Library Services (IMLS) que oferece, também, a possibilidade de comparação entre bibliotecas com outras variáveis. (INSTITUTE OF MUSEUM AND LIBRARY SERVICES IMLS).

Esse processo de divulgação da classificação das bibliotecas gera expectativas, atenção e espaço na mídia. Sobretudo porque as unidades agraciadas com cinco estrelas comemoram esse fato nas respectivas comunidades e empenham-se em divulgar os resultados na mídia/redes sociais. (LIBRARY..., 2015).

Cria-se, dessa forma, um ambiente de análise e comparação em que as bibliotecas são informadas das características dos processos exitosos e das experiências problemáticas a evitar. Estimula-se a concorrência e aprimora-se, assim, o sistema das bibliotecas públicas.

\subsubsection{Noruega}

Na Noruega, a Biblioteca Nacional da Noruega e a unidade de Estatísticas da Noruega (Kostra), criada em 2002, para informações municipais e distritais, coletam e publicam, anualmente, as estatísticas referentes às bibliotecas públicas. Em 2008, as Autoridades Norueguesas de Arquivos, Bibliotecas, Museus criaram os indicadores de avaliação.

A Kostra calcula e reproduz os indicadores para bibliotecas públicas desde 2009, para o universo de 430 unidades. São 13 indicadores: seis de produção; cinco dos recursos utilizados; dois de produtividade. São eles: custo corrente líquido das bibliotecas públicas em 
relação ao custo corrente total da municipalidade; custo corrente líquido das bibliotecas públicas per capita (Coroas);empréstimo de todas as mídias públicas (unidades físicas) per capita; empréstimo de livros (unidades físicas) per capita; número de livros emprestados per capita para crianças de 0-13 anos de idade; número de livros emprestados per capita para pessoas com mais de 14 anos de idade; empréstimos de outras mídias (unidades físicas) no total das bibliotecas pública per capita; consultas de livros infantis na biblioteca pública; consultas de livros de ficção para adultos na biblioteca pública; acréscimo de todas as mídias nas bibliotecas públicas por 1.000 habitantes; número de visitas nas bibliotecas públicas per capita; gastos com mídias e salários nas bibliotecas públicas per capita; e número de habitantes por funcionários (staff) trabalhando o equivalente a tempo integral nas bibliotecas públicas.

Os indicadores são também previstos para benchmarking no nível nacional e local. O propósito do benchmarking é encontrar áreas para melhorar os serviços e os processos, estudando as melhores práticas da área das bibliotecas. [...] Espera-se que muitas bibliotecas públicas comecem a utilizálos e ganhem experiência na aplicação desse processo. (REDSE; RA, 2011).

\subsubsection{Suécia}

Todo ano, o Conselho Sueco de Artes coleta, trata e edita as estatísticas das bibliotecas do setor público. A Associação das Bibliotecas Suecas elabora 10 indicadores anualmente, por sua própria inciativa, baseados nas estatísticas do Conselho. (LINDMARK, 2010). São eles: número de visitas per capita; total de empréstimos per capita; número de livros infantis emprestados por criança de 0-14 anos; número de livros infantis por criança de 0-14 anos; número de livros per capita; novas aquisições per capita; coleções de audiovisuais per capita; assinatura de revistas por mil habitantes; número de empregados por 1.000 habitantes; e custo operacional total per capita.

As avaliações dos resultados comparativos orientam as políticas de distribuição de recursos: "Muitas municipalidades percebem que a alocação dos fundos é baseada somente em alguns desses indicadores. Fazem uma análise do desempenho das bibliotecas baseada nos indicadores em que o valor maior é compreendido como melhor". (LINDMARK, 2010).

\subsubsection{Dinamarca}

Anualmente, a Denmark Statistic divulga os dados referentes às bibliotecas públicas. A Agência Dinamarquesa para Bibliotecas e Mídia em colaboração com a Gentofte Library contratou, em 2007, a firma Key Performance Index (KPI), para desenvolver um sistema de quantificação 
das visitas digitais e utilização dos sites das bibliotecas públicas. (KVIST, 2011).

A principal preocupação é tornar comparáveis os valores entre as bibliotecas, criando, assim, uma referência. Os dados do site de uma biblioteca individual não têm valor por si mesmos porque é impossível saber se 10.000 visitas por mês é muito ou não. (KVIST, 2011).

Desse sistema, foram selecionados ainda alguns indicadores, denominados índice dinamarquês de bibliotecas, publicados semanalmente: número de páginas vistas, visitas, visitantes e visitas por mil habitantes. (DANMARKSBIBLIOTEKSINDEX).

Angermann (2011) observa:

As atividades 'online' das bibliotecas públicas têm mudado consideravelmente desde o estabelecimento do índice de bibliotecas em 2009. Cada vez mais as bibliotecas estão usando a internet de forma mais versátil e direcionando comunicações e serviços para certos específicos grupos, necessidades e situações.

Em 2011, iniciou-se outro levantamento nacional nas bibliotecas públicas dinamarquesas, buscando medir a satisfação dos clientes. A iniciativa possibilita a comparação entre elas mesmas em períodos diferentes e entre as demais bibliotecas de tamanho compatível. Coordenam o levantamento a Herning Library e a empresa Ramboll a cada dois anos. (SVENSTRUP, 2011, p. 19).

Esses sistemas de coleta de dados preenchem as precondições que facilitarão, possivelmente, a implantação de um processo de benchmarking no futuro.

\subsubsection{Outros países}

No âmbito internacional, a Seção Metropolitana das Bibliotecas (MSL) da International Federation of Library Association (IFLA) realiza esse tipo de avaliação desde 2000. São pesquisadas 56 bibliotecas da Ásia, Europa, América do Norte e Oceania. A coleta de dados possibilita a publicação de 20 indicadores. ( DI TILLIO, 2013). Os dados mais recentes encontram-se disponíveis no site da IFLA MLS (2012). Os dados são compilados por diferentes bibliotecas participantes em diferentes períodos. Por exemplo, em 2012, na Austrália e, em 2011, na Finlândia. As unidades encontram-se ranqueadas no site da IFLA.

Allison Dobbie do Conselho de Auckland/Australia afirma que "usou as estatísticas como benchmark para verificar os níveis de recursos alocados em relação com os níveis das outras bibliotecas de tamanho similar. Foi muito útil para justificar os níveis de recursos para o nosso conselho". (DI TILLIO, 2013).

No caso brasileiro a gestão da informação do Sistema Nacional de Bibliotecas Públicas (SNBP), ou seja, coleta, análise, divulgação podem ser 
assim resumida: as bibliotecas públicas municipais (BPMs) preenchem os relatórios/formulários (frequência, consulta) trimestralmente e encaminham para a biblioteca estadual onde os dados são consolidados e arquivados. $^{3}$ Alguns estados e municípios, já disponibilizam essas informações, por exemplo, a Prefeitura da Cidade de São Paulo (SÃO PAULO). Recentemente, foi criado um portal pelo SNBP para ser voluntariamente atualizado/abastecido, com os dados supracitados, pelas BPMs (vide site SNBP)). Pesquisas realizadas pela equipe mostraram que o processo encontra-se com relação à disponibilidade de dados, ainda numa fase inicial. Uma visão/compreensão do SNBP é apresentada por Machado (2010); Silva (2013); e a Fundação Pensamento Digital (2013).

As precondições para estimular e fomentar o processo de benchmarking no âmbito estadual e municipal acredita-se que a situação atual atenderia parcialmente tais condições conforme análise de Maciel Filho et al. (2004). Em nível nacional, no entanto, encontram-se, do ponto de vista operacional, como visto acima, mais restritas.

A quantidade de indicadores dos diversos países varia: a Alemanha utiliza 18; a Suécia, 10; Noruega, 13; Estados Unidos, 4; IFLA, 20; e a Dinamarca 4. Assim, poder-se-ia começar a examinar qual seria 0 número de indicadores mais eficiente. A avaliação requereria uma análise dos resultados alcançados versus os custos do programa. Acreditase, no entanto, que a experiência na utilização dos indicadores nos processos comparativos poderia sinalizar as mudanças necessárias para propiciar o aprimoramento das bibliotecas dentro de uma estrutura de custo sustentável, ou seja, um benchmarking entre sistemas buscando um melhor custo/benefício.

\subsection{Outros estudos sobre benchmarking com amostras de menor dimensão}

Poll (2007, p. 43) analisou indicadores de bibliotecas públicas de diversos países: Alemanha (Bix- bibliotecas públicas), Austrália (bibliotecas públicas), Reino Unido (acadêmicas, 174 ), Suécia (diversos tipos, 60) e Holanda (acadêmicas, 14).

$\mathrm{Na}$ seção O benchmarking vale a pena o esforço, o autor analisa as grandes vantagens para os participantes do benchmarking e aponta alguns problemas a serem evitados/ administrados, ou seja, os efeitos colaterais não antecipados. (POLL, 2007, p. 51).

As vantagens internas são: a possiblidade de valorar seus próprios resultados por comparação; ajuda para identificar os problemas nos processos na própria organização; possibilidade de compartilhar experiências com as bibliotecas as melhores práticas de gestão; e maior percepção e aceitação das avaliações e dos procedimentos de controles realizados nas bibliotecas.

\footnotetext{
${ }^{3}$ Informação prestada por Maria Lúcia Ferreira, bibliotecária da Biblioteca Pública do Estado de Pernambuco, em 15 de fevereiro de 2016.
} 
Constituem vantagens para divulgações externas das bibliotecas: transparência dos gastos realizados e da qualidade alcançada; aumento da atenção para as bibliotecas e seus serviços pela apresentação dos resultados; e aumento da credibilidade dos relatórios das bibliotecas, propiciando uma garantia de neutralidade.

Dificuldades que precisam ser avaliadas: a necessidade de um controle central do processo para padronizar os dados coletados, evitando-se comparações enganosas; a divulgação pode prejudicar as bibliotecas com baixa avaliação, como também as mais bem avaliadas que podem vir a perder recursos oriundos das mantenedoras; a possibilidade de gerar comportamentos distorcidos nas suas atividades, procurando alcançar uma avaliação mais elevada no curto prazo e, assim, adiando decisões mais importantes; e a participação voluntária é problemática, visto que, a mudança constante das instituições participantes pode afetar a comparação de resultados ao longo dos anos.

Depois de três anos, o projeto de benchmarking das bibliotecas holandesas apresentou as experiências, assim sumarizadas ${ }^{4}$ por Laeven e Smit ( 2003 apud POLL, 2007, p. 51)

Nós concluímos dizendo que o desenvolvimento do sistema de benchmarking não é um pequeno projeto e os instrumentos usados pelas bibliotecas holandesas ainda estão longe de serem perfeitos, mas na nossa visão, o valor do benchmarking como uma comprovada ferramenta para alcançar qualidade na administração deve ser valorada como extremamente alta com certeza.

Kortelainen (2007) analisa a aplicação da técnica de benchmarking em um grupo de 15 bibliotecas do norte da Finlândia em vários aspectos, como por exemplo, de desempenho, de processos e de estratégias. Contudo, a ênfase principal é no benchmarking informal, com foco no que ele denomina partilhando conhecimentos e práticas (sharing knowledge and pratices).

A ideia é formar uma comunidade de bibliotecas onde as melhores práticas e os conhecimentos fossem compartilhados. Kortelainen (2007, p.105) destaca a importância da transferência de informação: "Partilhar conhecimento é uma parte crucial na inovação e no desenvolvimento, não somente na inovação industrial, como também em qualquer área do setor público e em numerosas diferentes tarefas".

O conceito de benchmark utilizado: "é uma ferramenta para melhoramento, alcançado por meio de comparação com outras organizações reconhecidas ${ }^{5}$ (CROSS; IABAL,1995 apud KORTELAINEN,

\footnotetext{
${ }^{4}$ LAEVEN, H.; SMIT, A. A project to benchmark university libraries in The Netherlands. Library Management, v. 24, p. 291-304, 2003. Disponível em: <www.ukb.nl/benchmark.htm>. Acesso em: 26 jul. 2015.

CROSS, R.; IABAL, A. The Rank Xerox Experience: benchmarking ten years on. In: ROLSTADÅS, A. (Ed.) Benchmarking: theory and practice. London: Chapman \& Hall, 1995.
} 
2007, p. 106) como a melhor dentro da área ou por meio de um relativo ótimo local ${ }^{6}$. (KOUZMIN et al., 1999 apud KORTELAINEN, 1999, p. 106).

O projeto durou três anos. Os resultados alcançados, após dois anos de seu término, assim podem ser resumidos: "É possível ver que novas práticas foram adotadas nos procedimentos/processos das bibliotecas estudadas". (KORTELAINEN, 2007, p. 110).

Em 2004, realizou-se um estudo sobre a potencialidade dos ganhos decorrentes da utilização do benchmarking no Sistema de Bibliotecas Públicas Municipais (SBPM) do Estado de Pernambuco. (MACIEL FILHO et al., 2004). Analisaram-se 176 unidades no período de cinco anos, entre 1998-2002. Cabe salientar que os autores e os estagiários/bolsistas do projeto precisaram digitalizar os dados. Construíram-se alguns indicadores de desempenho e, com o auxílio destes, identificaram-se as melhores práticas de gestão (best pratices). Estimou-se, também, o potencial de aumento nos serviços prestados, caso as unidades conseguissem alcançar o desempenho médio ou, ainda, a média das dez melhores BPMs, ou seja, as experiências exitosas. Sugeriram-se, também, políticas buscando auxiliar as BPMs a melhorarem os respectivos desempenhos com 0 compartilhamento das melhores práticas (best pratices).

Os estudos analisados, de um modo geral, evidenciaram um potencial de ganhos elevados caso fossem compartilhadas as práticas e os conhecimentos obtidos/identificados nas experiências exitosas.

\subsection{Projetos especiais}

O projeto Iniciativa Edge, liderado pelo The Urban Library Council (ULC/ USA), proporciona uma autoavaliação, uma ferramenta de benchmarking e outros recursos para assistir às bibliotecas públicas e para avaliar e melhorar os serviços digitais. (EDGE).

As participações das bibliotecas possibilitam uma melhor compreensão da produção (output), como também dos resultados(outcome). Esse projeto trabalha simultaneamente com a pesquisa de impacto. Ambos foram financiados pela Gates- Foundation.

O objetivo é informar os administradores das bibliotecas públicas e partes interessadas que querem compreender como e para que propósito os usuários das bibliotecas usam os recursos digitais e serviços. (LYONS; LANCE, 2014, p. 27).

A Iniciativa Edge avalia os serviços tecnológicos das bibliotecas públicas. Com essa ferramenta as bibliotecas compreendem como funcionam as melhores práticas relativas aos serviços de tecnologia para a sua comunidade e determinam quais os passos necessários para melhorá-los.

\footnotetext{
${ }^{6}$ KOUMZMIM, A. et al. Benchmarking and performance measurement in public sectors: towards learning for agency effectiveness. International Journal of Public Sector Management, v. 12, n. 2, p. 121, 1999.
} 
Entre junho e setembro de 2013, sete sistemas de bibliotecas estaduais dos Estados Unidos, com um total de 160 bibliotecas, participaram do lançamento experimental da Iniciativa Edge. No fim de 2014, quase $30 \%$ das 9.244 bibliotecas, 2.773 públicas estavam registradas e 20 tinham completado o processo de avaliação. O projeto Edge é bem recente, porém, pelo número de bibliotecas que o utiliza é provável que o benchmarking já deva ter contribuído substancialmente para a gestão dessas unidades ${ }^{7}$.

\section{Bibliotecas Indústria do Conhecimento}

As Bibliotecas Indústria do Conhecimento (IDCs) são unidades desenvolvidas pelo Sesi, com o objetivo de promover a inclusão social e digital e facilitar o acesso à informação e à leitura para os munícipes e operários das indústrias. As unidades são implantadas pelo Sesi com a colaboração de parcerias locais, no caso de prefeitura, IDC Pública, se indústria, IDC Indústria, que se responsabilizam pela cessão do terreno e manutenção das instalações físicas. O tamanho padrão das IDCs é de $100 \mathrm{~m}^{2}$ e com um acervo médio de 1.300 títulos. Oferecem também 10 PCs com acesso à internet. No Brasil hoje, encontram-se funcionando 326 unidades e aguardando para serem inauguradas, 46 IDCs. $^{8}$ Elas não fazem parte do Sistema de Bibliotecas Públicas Municipais (SBPM), no entanto, desempenham, funções semelhantes às BPMs.

\subsection{Metodologia}

As respectivas unidades do Sesi cederam gentilmente os dados das IDCs de Alagoas (25), Paraíba (10), Rio Grande do Norte (10) e Pernambuco (18). As estatísticas não são totalmente compatíveis, o que exigiu algumas adaptações e estimativas de dados para complementar as séries anuais. Procurou-se utilizar os anos com maior número de informação mensal disponibilizada.

No presente estudo, analisam-se as IDCs Públicas. Para aplicar a metodologia de benchmarking, no primeiro momento, necessitou-se desenvolver índices, considerando a revisão da literatura supracitada, particularmente alguns indicadores utilizados pelo LJ, Bix, entre outros índices, e a disponibilidade de dados das IDCs. Os índices foram: de alcance municipal e o índice de eficiência. Posteriormente, foi necessário identificar as unidades com melhor desempenho (best practices) e hierarquizá-las.

Constatou-se, no estudo realizado em 2012, que $84 \%$ dos usuários das IDCs têm entre 0 e 18 anos no Estado de Pernambuco (MACIEL FILHO; ARAÚJO, 2012). Nesse sentido, assumiu-se que a população que frequentava as IDCs nos demais estados do Nordeste também era predominantemente constituída de jovens. Por conseguinte, escolheu-se para servir como base para medir o nível de alcance municipal das IDCs -

\footnotetext{
${ }^{7}$ Existe também uma experiência em Arapiraca/Alagoas visitada por um dos autores deste estudo.

${ }^{8}$ Dados recebidos por e-mail do Departamento Nacional do SESI, em 19 de janeiro de 2016.
} 
a população em idade escolar (PIE), que quantifica o número de habitantes do município com idade entre 4 e 17 anos.

O índice de alcance municipal é o resultado da divisão da quantidade de frequência pela PIE. Para tornar possível a comparação, elaborou-se um ranqueamento/hierarquização das IDCs em grupos compatíveis com a distribuição das PIE municipais por estado. O índice de eficiência é a relação entre o número de consultas/frequência mensal e o número de horas de funcionamento mensal das bibliotecas.

Os dados recebidos das IDCs se apresentavam sob diversas formas. Utilizou-se a variável com mais informação disponível e mais fácil de ser comparada: frequência.

Os dados recebidos do Sesi Alagoas foram: atendimento no computador, atendimento na biblioteca, educação continuada, trabalho da indústria e empréstimo. A variável frequência para as IDCs de Alagoas é igual à soma dos atendimentos no computador, na biblioteca, na educação continuada e no trabalho da indústria. Não se incluiu empréstimo porque se presumiu que é computado no total de atendimento da biblioteca, uma vez que o usuário pode utilizar o documento no recinto da biblioteca (consulta) ou no domicílio (empréstimo). Ao incluir os dados de empréstimo poder-se-ia estar contando o atendimento duas vezes. Eis as 25 unidades investigadas: Coruripe, São Miguel, Teotônio Vilela, Viçosa, Olho d'Água do Casado, Paulo Jacinto, São José da Tapera, Marechal Deodoro, Palmeira dos Índios, Piranhas, Atalaia, Arapiraca, Roteiro, Mar Vermelho, Batalha, Jequiá da Praia, Chã Preta, Maceió, São José da Laje, Cajueiro, União dos Palmares, Penedo, Santana do Ipanema, Maragogi e Água Branca.

Os dados das IDCs de Pernambuco recebidos foram: consulta e acesso. Considerou-se, como frequência, o acesso que segundo as técnicas do Sesi- PE Ana Paula e Alessandra, o acesso seria o equivalente à frequência. Oriundos de 18 unidades: Itaíba, Itapissuma, Bonito, Chã de Alegria, Agrestina, São João, Bezerros, Araripina, São Caetano, Limoeiro, Poção, Passira, Panelas, Nazaré, Santa Cruz, Tamandaré, Garanhuns e Floresta.

Os dados das IDCs da Paraíba foram: consulta ao acervo, pessoas atendidas, e empréstimo do acervo. Utilizou-se a variável pessoas atendidas como mais representativa da frequência. Eis as 10 unidades pesquisadas: Praça da Paz/João Pessoa, Parque das Crianças/Campina Grande, Praça Alcides Carneiro/Patos, Sousa, Cajazeiras, Praça Novo Milênio/Guarabira, Queimadas, Praça Nilo Feitosa/Monteiro, Itaporanga e Araruna.

Os dados recebidos das IDCS do Rio Grande do Norte estavam agregados por biblioteca e ano. No entanto, alguns relatórios mensais recebidos e visitas às IDCs da cidade de Macaíba e Santo Antônio permitiram compreender que os valores apresentados eram a soma das seguintes variáveis: atividades de grupo, acessos à internet, consulta ao acervo, reuniões pedagógicas, educação permanente e trabalhos de grupos. Portanto, a variável frequência, neste caso, corresponde à soma de todas as variáveis supracitadas. Eis as IDCs analisadas (10): Jardim do 
Seridó, Macaíba, Santo Antônio, São Gonçalo do Amarante, Serra Negra do Norte, Mossoró, Martins, Almino Afonso, Assú e Macau.

\subsection{Análise dos resultados}

São de 2013 os dados das IDCs Públicas dos estados Pernambuco, Alagoas e Rio Grande do Norte e de 2012, os da Paraíba.

Tabela 1 - Frequência anual das IDCs Públicas

\begin{tabular}{|c|c|c|c|c|c|}
\hline Estados & Média & $\mathrm{DP}^{*}$ & Média - $5^{\star \star}$ & Melhor Prática & Município MP \\
\hline Pernambuco & $13.560,72$ & $4.850,70$ & $18.595,00$ & $26.590,00 \mathrm{Fl}$ & Floresta \\
\hline Alagoas & $8.020,09^{* * *}$ & $7.887,37$ & $17.089,12$ & $44.713,09$ & Coruripe \\
\hline Paraíba & $5.750,37$ & $3.058,55$ & $8.317,04$ & $12.600,00$ & Parque das crianças \\
\hline RG Norte & $5.108,00$ & $2.546,80$ & $7.339,49$. & $9.065,00$ & Martins \\
\hline
\end{tabular}

Fonte: Dados da pesquisa.

$*_{\mathrm{DP}}=$ Desvio padrão MP $=$ Melhor prática $* *$ Media $5=$ média das frequências das as cinco melhores IDC.

***Sem Coruripe média 6.491,00 (DP 2523,14) e a média das 5 maiores 10183,125.

Os dados na Tabela 1 mostram uma variação acentuada com relação às frequências observadas entre as IDCs de Pernambuco. A média das cinco melhores alcança um valor superior à média geral em $50 \%$. A frequência na IDC de Floresta alcança o dobro da média. Isso pode se vê, também, no valor do desvio padrão (DP). Esse mesmo desempenho se observa nos estados da Paraíba e Rio Grande do Norte, ou seja, a relação entre a média e a média das cinco melhores e a unidade com o melhor nível de atendimento. Em relação às de Alagoas, as diferenças são mais acentuadas no caso da IDC de Coruripe. Excluindo-se essa IDC, a variação baixa de $7.887,37$ para $2.523,14$ e a média para 10.183,125. De uma forma geral, a diferença média entre a média estadual e a média das cinco melhores encontra-se em torno de $66 \%$ (retirando Cururipe da amostra esse valor é de 44\%).

Calculou-se, também, o percentual de frequência dos munícipes às IDCs, utilizando o índice de alcance municipal. Duas observações são pertinentes: as frequências não são individualizadas, ou seja, a mesma pessoa pode ir diversas vezes à mesma IDC e cada ida corresponde a uma frequência; e os municípios foram hierarquizados de acordo com suas PIEs para tornar mais comparável o desempenho das unidades.

Tabela 2 - Percentual de frequência em relação ao PIE- IDCs Públicas

\begin{tabular}{llcccccccc}
\hline & & PE & \multicolumn{3}{c}{ AL } & \multicolumn{3}{c}{ PB } & \multicolumn{3}{c}{ RN } \\
\hline Cidades & PIE & M & MP & M & MP & M & MP & M & MP \\
\hline Grupo 1 & $>15000$ & 0,49 & 0,56 & 0,40 & 2,41 & 0,17 & 0,30 & 0,20 & 0,3 \\
Grupo2 & $10000-15000$ & 0,78 & 0,91 & 0,50 & 0,72 & 0,26 & 0,49 & 0,36 & 0,36 \\
Grupo 3 & $5000-10000$ & 1,68 & 3,02 & 0,65 & 1,11 & 0,85 & 1,34 & 0,45 & 0,61 \\
Grupo 4 & $<5000$ & 2,09 & 2,09 & 2,48 & 4,98 & & & 2,34 & 3,85 \\
\hline
\end{tabular}

Fonte: Dados da pesquisa.

M- Média; MP- melhor prática municipal de cada estado. 
A Tabela 2 mostra que a frequência é bastante significativa nas IDCs do grupo 4, onde a PIE é inferior a 5.000 mil habitantes. Isso significa que, em média, atendem mais que a população em idade escolar por ano. Por exemplo, Alagoas 2,48 vezes a mencionada população; Rio Grande do Norte 2,34 e Pernambuco 2,09. Também se visualiza que alguns estados apresentam uma diferença maior entre a média estadual e a melhor prática. Por exemplo, para alguns grupos: Pernambuco no grupo 3, Alagoas no grupo 1, Paraíba nos grupos 1 e 2 .

Observa-se, ainda, que à medida que a população total cresce, o percentual de frequência em relação ao PIE diminui. Esse fato pode decorrer das características das IDCs, em que as unidades têm a mesma dimensão física com pequenas variações no acervo e número de pessoas que nelas atuam, ou seja, semelhante aos rendimentos decrescentes. Ou, ainda, da existência de bibliotecas escolares nos municípios mais populosos.

Quanto aos 16\% restantes (MACIEL FILHO; ARAÚJO, 2012), dividiu-se pela população alfabetizada acima de 15 anos (IBGE, 2010). Os cálculos mostraram que para os municípios na faixa de 0-5.000 habitantes, em PE e $A L$, o índice de alcance municipal correspondeu a $45 \%$ da mencionada população.

Prosseguindo-se na análise, calculou-se o índice de eficiência.

Tabela 3- Índice de eficiência -número de frequência por hora- IDCs Públicas

\begin{tabular}{lllccl}
\hline \multicolumn{1}{c}{ Estados } & IE & DP & IE5 & Mais eficiente & Município \\
\hline Pernambuco & 4,7 & 2,7 & 8,0 & 12,6 & Floresta \\
Alagoas & 2,7 & 3,0 & 7,1 & 5,0 & São Miguel dos Campos \\
Paraíba & 2,5 & 1,4 & 3,3 & 5,3 & Parque da Criança
\end{tabular}

Fonte: Dados da pesquisa.

IE = Índice de eficiência médio mensal; IE5 = Média dos indicadores das cinco mais eficientes unidades. DP = desvio padrão.

O valor de Cururipe $(A L)$ distorce as médias. Com Cururipe, a média é de 3,4 e o seu nível de eficiência é de16, 9. Na Tabela 2, os valores não incluem Coruripe. Observa-se na Tabela 3 que a frequência média por hora nas IDCs em Pernambuco é de aproximadamente 4,7 visitas. Verifica-se que as médias das cinco melhores IDCs representam $70 \%$ a maior desse valor $(8,01)$ e a IDC com a melhor prática representa 2,5 vezes esse valor, ou seja, 12,6. No caso da Paraíba, esse valor chega a 2,1 vezes $(5,3 / 2)$ e no de Alagoas 1,9 vezes.

\subsection{Discussão}

Os dados coletados permitiram uma análise da situação atual e possibilitaram também estimar o potencial de crescimento de frequência das IDCs. As IDCs prestam serviços a um percentual elevado da população em idade escolar. Nas cidades onde a população em idade 
escolar é inferior a 5.000, o número de visitas chega ao equivalente a mais de duas vezes dessa população no ano.

Os dados evidenciam a variação da frequência entre as IDCs de PE, $A L, P B$ e RN. Acontece tanto do ponto de vista de alcance municipal, quantidade de idas da população em idade escolar às IDCs, como também de eficiência, ou seja, número de frequência por hora.

As IDCs são fisicamente iguais. Existem pequenas variações: no acervo e na quantidade de pessoas que nelas atuam; além das características específicas de cada comunidade; e da localização dentro de cada cidade. Uma unidade central coordena o projeto em cada estado e uma, nacionalmente. As estatísticas das IDCs estão disponíveis para seus gestores locais e estaduais.

Os dados permitem ainda efetuar simulações buscando-se visualizar o potencial de ganhos provenientes do uso do benchmarking.

Utilizando-se as estatísticas do estudo de caso, poder-se-iam implementar estratégias para aumentar frequência, visando-se elevar as visitas ao equivalente às médias das cinco melhores IDCs dos quatro estados investigados: AL alcançaria 56\% de aumento (ou 88.000 visitas adicionais para 24 unidades ); PE, 38,5\% ( 87.951 visitas para 17 unid.); PB, $45 \%$ (ou 25.667 para 10 unid.); RN, 44\% (22.314 vistas para 10 unid.). Essas metas podem parecer elevadas, no entanto, é bom recordar que as unidades são fisicamente iguais.

É importante relembrar que outros segmentos das comunidades também visitam as IDCs como, por exemplo: trabalhadores, aposentados, donas de casa, alunos do terceiro grau, empreendedores, pequenos empresários entre outras categorias.

Os indicadores desenvolvidos possibilitariam as seguintes sugestões relativas aos índices de alcance municipal e de eficiência.

$O$ índice de alcance indica quais IDCs poderiam estimular a frequência em relação à população em idade escolar. Orientaria políticas de aproximação com as escolas públicas, de implantação de novas atividades, de maior divulgação das já existentes junto à comunidade e de ampliação do horário de atendimento.

O índice de eficiência mostra a possibilidade de aumentar o número de visitas por hora. Verificar-se-iam os horários de menor utilização para desenvolver atividades com diferentes grupos do alunado, como por exemplo: direcionar para as crianças de dois a cinco anos os horários de menor afluência do alunado do ensino fundamental I e II ou para os alunos do ensino médio. Incentivar, também, o uso por outros segmentos da comunidade, como idosos/aposentados e demais trabalhadores ou outras para atividades, como por exemplo, aulas de matemática com auxílio do site da Academia de Khan (KAHANACADEMY).

As opções de políticas são múltiplas. As experiências exitosas das melhores práticas serviriam de orientação e ofereceriam parâmetros para tomada de decisão. Por exemplo, o Sesi de Pernambuco em 2015 adota a prática de reunir duas vezes por ano os que atuam nas IDCs em cada uma das microrregiões para troca de experiência e informações. 
A execução de políticas de benchmarking no sistema das IDCs não é uma tarefa difícil, tendo em vista as precondições nele existente; compõeno aproximadamente 326 unidades (utilizando para o Brasil, a proporção observada nos quatros estados pesquisados, se teriam 271 IDCs Públicas e 55 IDCs Indústrias). No entanto, é necessário observar as seguintes etapas:
a)sistematizar/ padronizar e unificar os conceitos relativos às informações coletadas;
b)aprimorar a coleta de dados;
c) estabelecer indicadores de benchmarking ${ }^{9}$;
d)ranquear e identificar as IDCs com melhor desempenho;
e)analisar as práticas / experiências exitosas utilizadas por essas unidades;
f) divulgá-las;

g)criar sistema / mecanismos para divulgá-las e compartilhá-las com as IDCs do mesmo estado e entre eles;

h)utilizar mais e melhor os diversos canais de comunicação (internet, conferências virtuais, reuniões, encontros); e

i) envolver mais e melhor as comunidades beneficiadas.

A estratégia de benchmarking a ser adotada seguiria Kortelainen (2007, p. 105), ou seja, "formar uma comunidade de bibliotecas onde as melhores práticas e os conhecimentos fossem compartilhados".

\subsubsection{Outras observações}

Algumas sugestões da literatura recente dão ênfase à contribuição das bibliotecas para o desenvolvimento econômico da comunidade em que estão inseridas e que poderiam aumentar as frequências às IDCs.

Destaca-se a importância de atender/oferecer serviços às crianças da faixa de até cinco anos. De acordo com o professor Heckman (2008), premiado com o Nobel em economia, seria a idade em que o investimento em educação, proporcionalmente, teria o maior retorno para sociedade (Gráfico 1), sobretudo para as crianças pobres, no sentido de reduzir os custos sociais futuros (reduzir encarceramentos, uso de drogas, abandono das escolas, gasto em saúde), e aumentar desempenho na escola, universidade, vida profissional (HECKMAN, 2016). As IDCS poderiam contribuir dando ênfase à literatura infantil, jogos e sobretudo oferecendo a hora do conto para as crianças menores de três anos, já oferecida em algumas IDCs e nas Arapiraquinhas - bibliotecas de Arapiraca - sem,

\footnotetext{
${ }^{9}$ É importante ressaltar que o SESI-PE já utiliza o ranqueamento das IDCs baseados em acessos e outras variáveis. Poderia, desta forma, com facilidade, adaptar/desenvolver outros indicadores e utilizá-los em comum acordo com outros Sesis estaduais.
} 
contudo, ser o foco nessa idade e desenvolver atividades atraindo/motivando os pais leitores.

Gráfico 1 - Retorno por dólar investido em capital humano

HECKMAN: SCHOOLS, SKILLS AND SYNAPSES

\section{Returns to a Unit Dollar Invested}

(a) Return to a Unit Dollar Invested at Different Ages from the Perspective of the Beginning of Life, Assuming One Dollar Initially Invested at Each Age

Programs targeted towards the earliest years

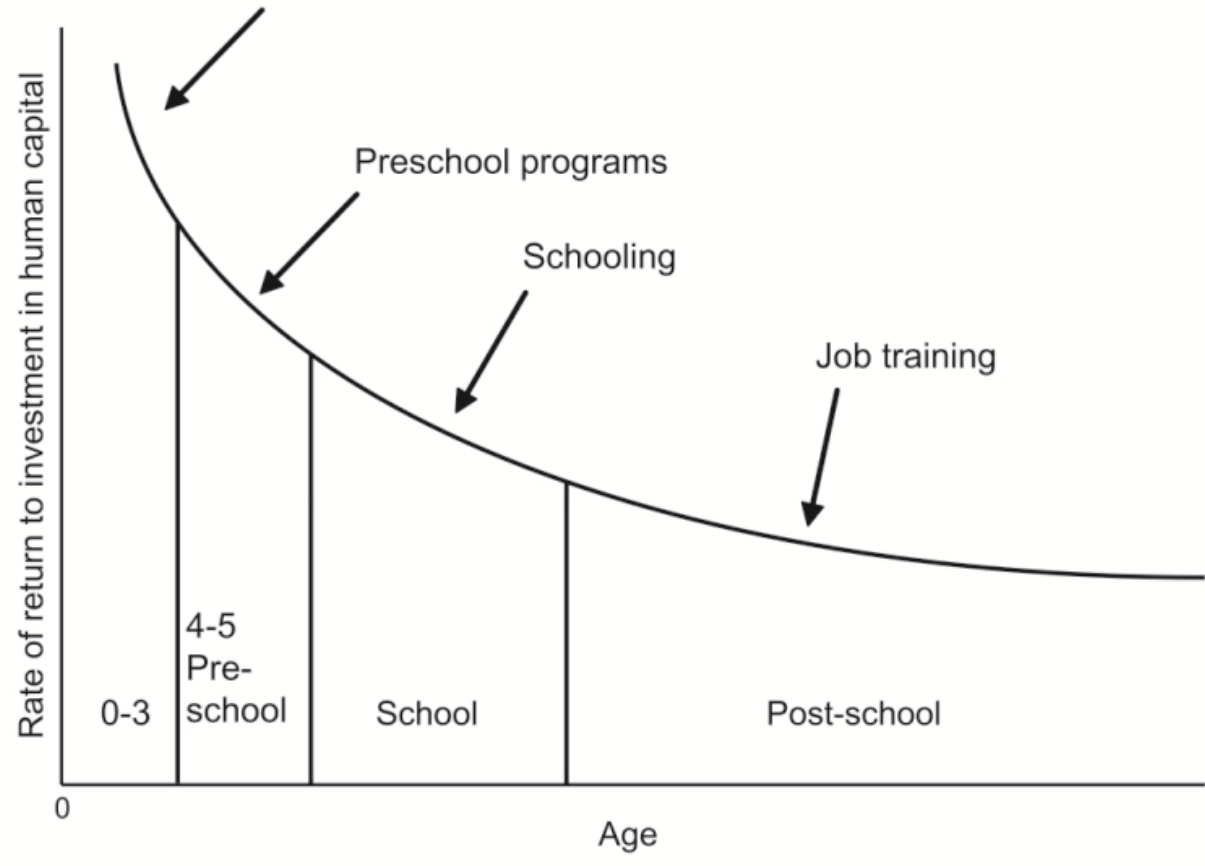

Fonte: HENCHMAN (2008).

É sugerido, ainda: disponibilizar a literatura e as demais informações necessárias ao aprimoramento/qualificação e autoestudo dos trabalhadores dos diversos setores, ou seja, apoiar o desenvolvimento da força de trabalho. (MACIEL FILHO et al., 2002; THE URBAN LIBRARY COUNCIL, 2007, p. 24-25); e propiciar acesso às informações pertinentes aos empreendedores das pequenas e microempresas dos diversos setores (THE URBAN LIBRARY COUNCIL, 2007, p. 24-25). Nesse sentido, existe a experiência nas IDCs de Pernambuco, onde o Sebrae (PE) oferece diversas coleções destinadas a esse público nas IDCs. (MACIEL FILHO; AQUINO; OLIVEIRA, 2008).

\section{Considerações Finais}

A técnica de benchmarking está bastante difundida nos setores público e privado nos mais diversos países. 
O ranqueamento/hierarquização das universidades americanas pela revista US News \& World Report propicia uma competição acentuada entre essas instituições. Nos programas de MBA, entre os cursos extremamente rentáveis, a concorrência para alcançar e permanecer entre as top 20 é elevada. O ranque da Bloomberg-businessweek é bastante utilizado. (RODKIN; LEVY, 2015).

A utilização do benchmarking pelo grupo privado dos empresários Paulo Lemann, Marcel Telles, Beto Sicupira, este ainda na fase inicial da expansão: "Nos primórdios, os três olharam do Brasil para os Estados Unidos e viram o que já funcionava. Então, em vez de aguardarem que aquilo chegasse ao Brasil, agiram agressivamente para importar as melhores práticas americanas antes dos outros". (CORREA, 2013, p. 11). Outro exemplo: "O Wal-Mart desempenhou para as Lojas Americanas o mesmo papel que o Goldman Sachs teve para o Garantia - era o modelo a ser copiado, a maior fonte de inspiração". (CORREA, 2013, p. 78).

$\mathrm{Na}$ Alemanha, o benchmarking foi contemplado na reforma constitucional para estabelecer a comparação entre os mais diversos serviços oferecidos pelo setor público. (KNUPLING, 2012, p. 11).

As diversas experiências internacionais, discutidas na segunda parte desse texto, mostram a amplitude da utilização do benchmarking nos sistemas de bibliotecas públicas dos diversos países. Outra linha de aplicação da técnica de benchmarking é apresentada por Carvalho et al. (2011).

Reafirma-se, no caso brasileiro, que a possibilidade de implantação de um processo de benchmarking nas bibliotecas públicas no âmbito nacional é mais restrita. No entanto, as precondições são parcialmente atendidas no âmbito das unidades da federação, no caso das públicas municipais.

Nesse sentido, é necessário iniciar um projeto para sistematizar as estatísticas disponíveis a partir de uma amostra significativa e, posteriormente, estabelecer os indicadores, hierarquização etc., seguindose a estratégia proposta na seção anterior. Uma vez aplicado em algumas unidades da federação, provavelmente, o processo será adotado pelas demais pelos bons resultados alcançados.

No caso das IDCs Públicas, as precondições existem. Necessita-se, portanto operacionalizar o processo de benchmarking de acordo com as etapas especificadas na Discussão. Essa ação poderá propiciar uma elevação na frequência das PIEs nas 271 comunidades contempladas com as IDCs, contribuindo sobretudo para desenvolver o hábito da leitura, e proporcionar os demais benefícios provenientes da utilização de bibliotecas. Dessa forma, apoiaria o sistema educacional que, de acordo com os resultados de português e matemática das últimas avaliações (PISA, 2013), está necessitando toda atenção possível. Outros segmentos da comunidade também poderiam ser contemplados com os demais serviços oferecidos pelas bibliotecas como foi mencionado na Discussão.

Tem-se, no presente momento, uma grande oportunidade de implantar um programa de grande alcance social. Os estudos empíricos baseados na economia comportamental mostram a importância de 
pequenos empurrões (nudge) que propiciam ganhos relevantes no desempenho institucional (WORLD BANK, 2015). O reconhecimento das melhores bibliotecas, o compartilhamento de informações e interação entre elas possibilitam melhorar a eficiência do sistema bibliotecário, seguindo-se assim o processo que vem sendo utilizado em diversos países.

\section{Referências}

ANGERMANN, T. Development of the library index. Scandinavian Libray Quarterly, v. 44, n. 1, 2011. Disponível em: <http://slq.nu/?article=denmark-development-of-the-library-index $>$.

Acesso em: 5 jul. 2015.

ASSOCIAÇÃO ALEMÃ DE BIBLIOTECAS; NORTH RHINE-WESTPHALIAN LIBRARY SERVICE CENTER. Disponível em: <http://www.bixbibliotheksindex.de/en/project-info.html>. Acesso em: 8 ago. 2015.

BIX Indicator for public library. Disponível em: <http://www.bixbibliotheksindex.de/en/ projectinfo/indikatoren/oeffentlichebibliotheken.html>. Acesso em: 3 ago. 2015.

CARVALHO, F. A. A. et al. Análise envoltória de dados na gestão do desempenho de bibliotecas universitárias: o caso de uma IFES no Rio de Janeiro. Revista Brasileira de Biblioteconomia e Documentação, Nova Série, São Paulo, v. 7, n. 1, p. 4-21, jan./jun. 2011.

CORREA, C. Sonho grande. Rio de Janeiro: Sextante, 2013.

CROSS, R.; IABAL, A. The Rank Xerox Experience: Benchmarking ten years on. In: ROLSTADÅS, Asbjorn (Ed.) Benchmarking: Theory and Practice. London: Chapman \& Hall, 1995.

DANMARKSBIBLIOTEKSINDEX. Disponível em: <danmarksbiblioteksindex.dk>. Acesso em: 10 nov. 2015.

DI TILLIO, C. A look at library data. Public Libraries on Line. 2013. Disponível em: <http://publiclibrariesonline.org/2013/12/a-look-atlibrary-data/>. Acesso em: 10 jun. 2015.

EDGE. Disponível em: <http://www.libraryedge.org/about-edge/history>. Acesso em: 20 jun. 2015.

FAVRET, L. Benchmarking, annual library plans and best value: the implications for public libraries. Library Management, Bradford, v. 21, n. 7, p. 340-348, 2000.

FENNA, A.; KNUPLING, F. (Eds.). Benchmarking in the federal systems. Camberra: Productivity Commission, 2012.

FUNDAÇÃO PENSAMENTO DIGITAL. Uso de tecnologias da informação e comunicação em bibliotecas públicas do Brasil: relatório de pesquisa. Porto Alegre, 2013. Disponível em: 
<http://ticsbps.weebly.com/uploads/2/1/0/4/21045142/

relatoriodepesquisa_ptg.pdf>. Acesso em: 17 jan. 2016.

HECKMAN, J. J. The Heckman equation. 2016. Disponível em: $<$ http://heckmanequation.org/content/resource/invest-early-childhooddevelopment-reduce-deficits-strengthen-economy>. Acesso em: 20 jan. 2016.

HECKMAN, J. J. Schools, skills, and synapses. Cambridge, Ma: National Bureau of Economic Research, 2008. (NBER Working Paper, n. 14064, June 2008).

INSTITUTO BRASILEIRO DE GEOGRAFIA E ESTATÍSTICA (IBGE). Censo demográfico 2010. Disponível em: <http://cod.ibge.gov.br/2A9Z>. Acesso em: 10 out. 2012.

INTERNATIONAL FEDERATION OF LIBRARY ASSOCIATIONS (IFLA). METROPOLITAN LIBRARIES SECTION (MLS). 2012 survey summary report. Disponível em: <http://www.countingopinions .com/pireports/report.php?c2a1bda00b52d9e81bee2d48bba81c99>

Acesso em: 2 out. 2015.

INSTITUTE OF MUSEUM AND LIBRARY SERVICES (IMLS). Disponível em: <http://www.imls.gov/research/library_services.aspx>. Acesso em: 5 ago. 2015.

KHANACADEMY. Disponível em: <https://pt.khanacademy.org/>. Acesso: 2 jan. 2012.

KNUPLING, F. Introduction. In: FENNA, A.; KNUPLING, F. (Eds.). Benchmarking in the federal systems. Camberra: Productivity Commission, 2012. p. 1-10.

KONZENDORF, G.; HATHAWA, R. Towards benchmarking in Germany. In: FENNA, A.; KNUPLING, F. (Eds.). Benchmarking in the federal systems. Camberra: Productivity Commission, 2012. p. 111-122.

KORTELAINEN, T. Consortium benchmarking between information services of municipal libraries. Information Sciences (Informacijos Mokslai), n. 40, p. 105-114, 2007.

KOUMZMIM, A. et al. Benchmarking and performance measurement in public sectors: towards learning for agency effectiveness. International Journal of Public Sector Management, v. 12, n.2, p.121. 1999.

KVIST, U. KPI Index K(ey) P(erformance) I(ndex). Scandinavian Library Quarterly, v. 44, n. 1, 2011. Disponível em: $<$ http://slq.nu/?article=denmark-kpi-index-key-performance-index $>$. Acesso em: 19 jul. 2015.

LAEVEN, H.; SMIT, A. A project to benchmark university libraries in The Netherlands. Library Management, v. 24, p. 291-304, 2003. Disponível em: <www.ukb.nl/benchmark.htm>. Acesso em: 26 jul. 2015. 
LIBRARY named as prestigious Five-Star Library by Library Journal for third consecutive year. Disponível em: <http://www.cincinnatilibrary.org/news/2015/fivestarlibrary.html>. Acesso em: 9 set. 2015.

LINDMARK, C. Sweden: measure correctly or do the right thing: a national perspective on statistics and indicators. Scadinavian Library Quarterly, v. 43, n. 4, 2010. Disponível em: <http://slq.nu/?article=sweden-measurecorrectly-or-do-the-right-thing-a-national-perspective-on-statistics-andindicators\#sthash.sxdE2VjA.dpuf>. Acesso em : 4 jul. 2015.

LYONS, R.; LANCE, K. C. The LJ Index of the public library. Library Journal, $2014 . \quad$ Disponível em: <http://lj.libraryjournal.com/2014/11/managing-libraries/lj-index/classof-2014/the-star-libraries-2014>. Acesso em: 10 set. 2015.

MACHADO, E. C. Análise de políticas públicas para bibliotecas no Brasil. Revista de Ciência da Informação e Documentação, v. 1, n. 1, p. 94-111, 2010.

MACIEL FILHO, A.; ARAúJO, M. A. Indústrias do Conhecimento: estudo de caso. Relatório de pesquisa UPE. Recife, 2012. [Não publicado].

MACIEL FILHO, A; AQUINO, M; OLIVEIRA, G. T. P. Concepção de projeto para implementação, operacionalização e manutenção de um sistema de interação entre o Sebrae e bibliotecas públicas municipais: Projeto Biblioteca \& Empresa (Convenio FCAP-UPE Sebrae -2007). Versão preliminar. Recife, 2008. [Não publicado].

MACIEL FILHO, A. et al. Biblioteca \& empresa: relatório final (Projeto o aprovado junto à FACEPE do edital de Políticas Públicas. PUBLIC, 20002001). Recife, 2002.

MACIEL FILHO, A. et al. As melhores práticas administrativas: uma investigação empírica do Sistema das Bibliotecas Públicas Municipais do Estado de Pernambuco. Informação \& Sociedade, v. 14, n. 1, p. 129-144, jan./jun. 2004.

MUNDT, S. BIX: the Bibliotheksindex: statistical benchmarking in German public libraries. In: HEANY, Michael (Ed.). The library statitistic for the twenty first century World. Munchen: Deutche Nationalibliothek, 2009. p. 188-196.

PISA: desempenho do Brasil piora em leitura e 'empanca' em ciências. Disponível em: <http://educacao.uol.com.br/noticias/2013/12/03/pisadesempenho-do-brasil-piora-em-leitura-e-empaca-em-ciencias.htm $>$.

Acesso em: 15 jan. 2016.

POLL, R. Benchmarking with quality indicators: national projects. Performance Measurement and Metrics, v. 8, n. 1, p. 41-53, 2007.

PRÖHL, M.; WINDAU, B. (Eds.). Betriebsvergleich an Öffentlichen

Bibliotheken. Teil 1: Empfehlungen und Arbeitsmaterialien für ein output- 
orientiertes Berichtswesen. Gütersloh, 1997a. v. 1.

PRÖHL, M.; WINDAU, B. (Eds.). Betriebsvergleich an Öffentlichen Bibliotheken. Teil 2: Meßergebnisse - Richtwerte Handlungsempfehlungen. Gütersloh, 1997b. v. 2.

REDSE, T.; RA, E. Norway indicators for Norwegian public libraries. Scandinavian library Quarterly, v. 44, n. 1, 2011. Disponível em: <http://slq.nu/?article=norway-indicators-for-norwegian-public-libraries >. Acesso em: 10 jul. 2015.

RODKIN, J.; LEVY, F. Best business school 2015: Bloomberg Businessweek. Disponivel em : <http://www.bloomberg.com/features/2015-bestbusiness-schools/>. Acesso em: 12 jan. 2016.

SÃO PAULO. Prefeitura da Cidade de São Paulo. Sistema municipal de bibliotecas.

Disponível

em: <http://www.prefeitura.sp.gov.br/cidade/secretarias/cultura/bibliotecas/in dex.php?p=13740>. Acesso em: 10 fev. 2016.

SILVA, V. B. Biblioteca pública brasileira: panorama, perspectivas e a situação do Distrito Federal. Brasília, 2013. Disponivel em: <http://repositorio.unb.br/bitstream /10482/14228/1/

2013_VanessaBarbosaSilva.pdf.> Acesso em: 10 fev. 2016.

SISTEMA NACIONAL DE BIBLIOTECAS PÚBLICAS (SNBP). Disponível em: $<$ http://bibliotecas.cultura.gov.br/busca/\# \#\%28global:

$\% 28$ enabled:\%28space:!t\%29,filterEntity:space\%29\%29>. Acesso em: 10 fev. 2016.

SVENSTRUP, S. Almost 100 satisfaction and so what? Scandinavia Public Library Quarterly, n. 1, p. 18-19, 2011.

THE URBAN LIBRARY COUNCIL. Making cities stronger: public library contributions to local economic development. 2007. Disponível em: <http://www.urban.org/sites/default/files/ alfresco/publicationpdfs/1001075-Making-Cities-Stronger.PDF>. Acesso em: 2 fev. 2014.

WORLD BANK. World development report 2015:mind, society, and behavior. Washington, DC: World Bank, 2015.

\section{Agradecimentos}

Às técnicas Elaine Lopes, Arnaldo Carvalho- Sesi-Alagoas; Nathalia Cruz (Sesi-Paraíba); Triana Albuquerque, Francineide da Silva (Sesi-Rio Grande do Norte). Aos profissionais do Sesi-Pernambuco: Michael Groarke, Bruna Santos, Ana Paula Diaz, pelas informações prestadas e dados disponibilizados que tornaram possível esse trabalho. À Dra. Veridiana Negrini (SNBP). Ao Prof. Luiz Márcio de Oliveira Assunção pela leitura crítica e sugestões. 